\title{
Defects Studies of ZnO Single Crystals Prepared by Various Techniques
}

\author{
F. LukÁČ ${ }^{a, *}$, J. ČížEK ${ }^{a}$, I. ProcházKa ${ }^{a}$, O. Melikhova ${ }^{a}$, W. AnWAnd ${ }^{b}$ And G. Brauer ${ }^{b}$ \\ ${ }^{a}$ Charles University in Prague, Faculty of Mathematics and Physics \\ V Holešovičkách 2, 18000 Praha 8, Czech Republic \\ ${ }^{b}$ Institut für Strahlenphysik, Forschungszentrum Dresden-Rossendorf, Postfach 510119, D-01314 Dresden, Germany
}

\begin{abstract}
The aim of the present work was a comparison of defects in $\mathrm{ZnO}$ crystals grown by various techniques available nowadays, namely hydrothermal growth, pressurized melt, Bridgman method growth and vapor phase growth. Positron annihilation spectroscopy was employed as a principal tool for characterization of defects in $\mathrm{ZnO}$ crystals grown by above mentioned various techniques. $\mathrm{ZnO}$ crystals can be divided into two groups: (i) hydrothermal grown crystals, which exhibit positron lifetime of 179-182 ps and (ii) $\mathrm{ZnO}$ crystals grown by the other techniques (pressurized melt, Bridgman method, vapor phase growth) which are characterized by the lower lifetimes falling in the range of 160-173 ps. Comparison of experimental data with ab initio theoretical calculations revealed that HT grown $\mathrm{ZnO}$ crystals contains $\mathrm{Zn}$ vacancies associated with hydrogen atom in a bond-centered site. On the other hand, $\mathrm{ZnO}$ crystals prepared by other techniques contain most probably stacking faults created by stresses induced by temperature gradients in the melt.
\end{abstract}

DOI: 10.12693/APhysPolA.125.748

PACS: $78.70 . \mathrm{Bj}, 61.72 . \mathrm{J}-$

\section{Introduction}

Zinc oxide $(\mathrm{ZnO})$ is a perspective wide band gap semiconductor for blue/UV light emitters, detectors, and optoelectronic devices [1]. Despite the great progress in growth of the high quality $\mathrm{ZnO}$ crystals [2], the preparation and characterization of $\mathrm{ZnO}$ single crystals is still a challenging task since various methods provide samples with various defects introduced by the method. A detailed characterization of these defects is crucial since they often strongly influence electrical, magnetic, and optical properties of $\mathrm{ZnO}$ crystals [3].

Positron lifetime (LT) spectroscopy [4] provides unique tools for characterization of point defects present in a material. In case of $\mathrm{ZnO}$, the $\mathrm{LT}$ results available in literature show different lifetimes for as-grown crystals from various suppliers [5-9]. There is also a controversy in the interpretation of LT results. Therefore, a systematic investigation of $\mathrm{ZnO}$ crystals prepared by various methods is desirable in order to clarify the kinds of defects introduced by different methods of crystal growth. These experimental results were compared with ab initio theoretical calculations of positron annihilation characteristics for various defect configurations in $\mathrm{ZnO}$-lattice performed in Ref. [8].

\section{Experimental}

Single crystals with dimensions of $10 \times 10 \times 0.5 \mathrm{~mm}^{3}$ have been investigated. Hydrothermal (HT) grown samples were supplied by MaTecK GmbH (Jülich), CrysTec GmbH (Berlin), University Wafers (South Boston/MA), MTI (Richmond/CA) and Altra (Atomergic Chemetals

*corresponding author; e-mail: frantisek.lukac@mff.cuni.cz
Corp., Farmingdale/NY). Pressurized melt (PM) grown crystals were fabricated by Cermet Inc. (Atlanta/GA) and crystals prepared by Bridgman growth (BG) method were grown at the Institut für Kristallzüchtung (IKZ, Berlin).

A digital LT spectrometer [10] with excellent time resolution of 145 ps (FWHM ${ }^{22} \mathrm{Na}$ ) was employed for the LT investigations. $\mathrm{A}^{22} \mathrm{Na}_{2} \mathrm{CO}_{3}$ positron source with activity of $1.5 \mathrm{MBq}$ deposited on a $2 \mu \mathrm{m}$ thick Mylar foil was sandwiched between a pair of $\mathrm{ZnO}$ crystals. At least $10^{7}$ positron annihilation events were accumulated in the LT spectra which were decomposed using a maximum likelihood based procedure [11]. The source contribution consisted of two weak components which come from positrons annihilated in the source spot and the covering Mylar foil and exhibit lifetimes of $\approx 368 \mathrm{ps}$ and $\approx 1.5 \mathrm{~ns}$ and intensities of $\approx 7 \%$ and $\approx 1 \%$, respectively.

Variable energy positron annihilation spectroscopy (VEPAS) investigations were performed on a magnetically guided variable energy slow positron beam SPONSOR [12] at Helmholtz Zentrum Dresden-Rossendorf. Energy of incident positrons was varied in the range from 0.03 to $36 \mathrm{keV}$. Doppler broadening of annihilation profile was measured by HPGe detector with energy resolution of $(1.09 \pm 0.01) \mathrm{keV}$ at $511 \mathrm{keV}$ and analyzed using $S$ and $W$ line shape parameters.

\section{Results and discussion}

Results of the LT investigations of the HT, PM, BG, and vapor phase (VP) grown $\mathrm{ZnO}$ single crystals obtained in this work and collected from literature are listed in Table. Each LT spectrum after the source contribution correction consisted of a single component only. The lifetimes measured on various $\mathrm{ZnO}$ crystals are plotted in Fig. 1. From inspection of Fig. 1, it becomes clear that the positron lifetimes fall into two groups: (i) HT grown 
samples exhibit higher positron lifetimes in the range of 179-182 ps, while (ii) ZnO crystals grown by the other techniques (PM, BG, VP) exhibit shorter lifetimes falling into the range of $160-173 \mathrm{ps}$. There is only one exception from this division: Sarkar et al. [7] reported the lifetime of 164 ps for a HT grown ZnO crystal supplied by the MTI (Richmond/CA). To clarify this inconsistency, we purchased a HT grown $\mathrm{ZnO}$ crystal fabricated by the MTI company and re-measured it in our laboratory. One can see in Table that this crystal exhibits the positron lifetime of $(179.6 \pm 0.5)$ ps which belongs to the group (i) and is consistent with the lifetimes measured in HT grown crystals in our laboratory and also by other authors [5, 6]. For this reason, the value of $164 \mathrm{ps}$ reported by Sarkar et al. [7] was not considered further in this work and is not plotted in Fig. 1.

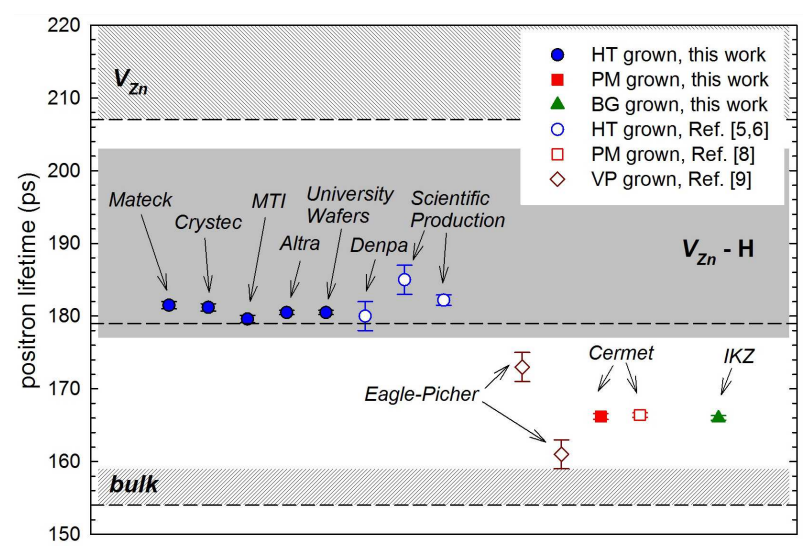

Fig. 1. Positron lifetimes measured in $\mathrm{ZnO}$ single crystals grown by various methods: HT, PM, BG, and VP growth. Results of $a b$ initio theoretical calculations of positron lifetimes for free positrons (bulk), positrons trapped at zinc vacancies $\left(\mathrm{V}_{\mathrm{Zn}_{\mathrm{n}}}\right)$, and positrons trapped at $\left(\mathrm{V}_{\mathrm{Zn}}\right)$ associated with hydrogen atom $\left(\mathrm{V}_{\mathrm{Zn}_{\mathrm{n}}}-\mathrm{H}\right)$ are shown in the figure as well. Shadowed bands show interval of positron lifetimes calculated within various theoretical approaches in Ref. [8]. Dashed horizontal lines show positron lifetimes calculated by the most accurate approach using the self-consistent electron density and considering ion relaxation around defects and positron induced forces.

In addition, Fig. 1 shows not only the experimental positron lifetimes but also the results of $a b$ initio theoretical calculations taken from Ref. [8]. The values of calculated positron lifetimes depend on the accuracy of the approach used in the calculations. The bands in Fig. 1 show the ranges into which fall positron lifetimes calculated using various approaches. Since the relaxations of the neighboring ions around the point defects are relatively large in $\mathrm{ZnO}$, we considered only the results of calculations obtained for relaxed defect geometries. The approach which uses the self-consistent electron density for calculation of a positron wave function and takes into account the forces imposed on ions by trapped positron can be considered as the most accurate one [8]. The positron
TABLE

Positron lifetime results for $\mathrm{ZnO}$ single crystals grown by various methods.

\begin{tabular}{c|c|c|c}
\hline \hline $\begin{array}{c}\text { Method } \\
\text { of growth }\end{array}$ & Supplier & $\tau_{1}[\mathrm{ps}]$ & Reference \\
\hline HT & MaTecK, Germany & $181.5(5)$ & this work \\
HT & Crystec, Germany & $181.2(5)$ & this work \\
HT & MTI, USA & $179.6(5)$ & this work \\
HT & Altra, USA & $180.5(3)$ & this work \\
HT & University wafers, USA & $180.5(3)$ & this work \\
HT & Denpa, Japan & $180(2)$ & {$[5]$} \\
HT & Scientific Production, & $185(2)$ & {$[5]$} \\
& Goodwill, Russia & & \\
HT & Scientific Production, & $182.2(7)$ & {$[6]$} \\
HT & Goodwill, Russia & $164(1)$ & {$[7]$} \\
PM & MTI, USA & $166.2(4)$ & this work \\
PM & Cermet, USA & $166.4(3)$ & {$[8]$} \\
BG & Cermet, USA & $166.0(3)$ & this work \\
VP & IKZ, Germany & $173(2)$ & {$[9]$} \\
VP & Eagle-Picher, USA & $161(2)$ & {$[9]$} \\
& & &
\end{tabular}

lifetimes calculated within this approach are plotted by dashed lines in Fig. 1. The bulk lifetime, i.e. the lifetime of free positrons in a perfect $\mathrm{ZnO}$ crystal, calculated using self-consistent electron density is 154 ps [8]. Obviously, this value is lower than the experimental positron lifetimes measured in $\mathrm{ZnO}$ crystals and listed in Table. It testifies that the most of the positrons are trapped at defects and the free positron component is so weak that it cannot be resolved in LT spectra (saturated trapping).

The theoretical calculations of point defects in $\mathrm{ZnO}$ available in literature consistently show that an oxygen vacancy is too shallow trap unable to confine positrons $[8,13,14]$. On the other hand, a zinc vacancy is a deep positron trap and defects found in the $\mathrm{ZnO}$ crystals should be therefore related to zinc vacancies. However, the lifetime of positrons trapped at zinc vacancy calculated using the self-consistent electron density and taking into account the ion relaxations and positron induced forces is $207 \mathrm{ps}$ [8] and the lifetimes calculated by other less accurate approaches are even higher, see Fig. 1. Hence, the lifetimes measured in as-grown $\mathrm{ZnO}$ single crystals are remarkably smaller than those calculated for the zinc vacancy. This can be caused by impurities bound to zinc vacancies which cause a shortening of the lifetime of trapped positron. In Refs. [8, 15] it was shown that hydrogen is the most important impurity in $\mathrm{ZnO}$ crystals and its concentration in $\mathrm{ZnO}$ crystals is more than one order of magnitude higher than the concentration of any other impurity. Moreover, hydrogen atoms are attracted to open-volume defects and form complexes with zinc vacancies $\left(\mathrm{V}_{\mathrm{Zn}}-\mathrm{H}\right)$. The lifetime of the positrons trapped at $\left(\mathrm{V}_{\mathrm{Zn}}-\mathrm{H}\right)$ complexes calculated in Ref. [8] using the self-consistent electron density and taking into account the ion relaxations and positron induced forces is $179 \mathrm{ps}$, see dashed line in Fig. 1, which agrees well with the life- 
times measured in HT grown $\mathrm{ZnO}$ crystals. ZnO crystals grown by other techniques exhibit shorter positron lifetimes which are, however, still higher than the $\mathrm{ZnO}$ bulk lifetime. Hence, in the $\mathrm{ZnO}$ crystals grown by the other techniques, the positrons are trapped at the defects with smaller open volume than the $\left(\mathrm{V}_{\mathrm{Zn}}-\mathrm{H}\right)$ complexes. Since a high density of stacking faults (SF) was found in $\mathrm{BG}$ grown $\mathrm{ZnO}$ crystals by X-ray diffraction combined with transmission electron microscopy, it has been suggested that in the $\mathrm{ZnO}$ crystals grown by $\mathrm{BG}$ and $\mathrm{PM}$ techniques positrons are trapped at open-volume misfit defects associated with stacking faults [15]. High density of SFs in these crystals can be attributed to thermal gradients existing in the melt.

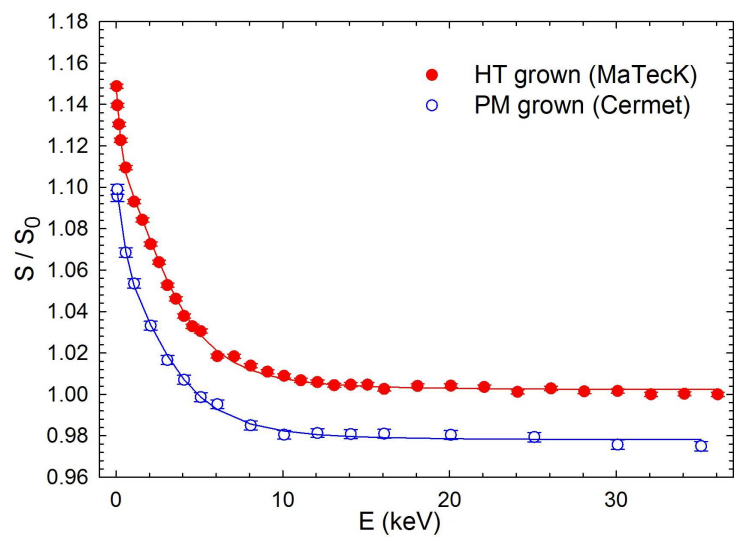

Fig. 2. VEPAS results: dependence of the $S$-parameter on the energy $E$ for HT (full points) and PM (open points) grown $\mathrm{ZnO}$ crystals. Model curves calculated by VEPFIT [16] are plotted by solid lines.

Figure 2 shows dependences of the $S$-parameter on the energy $E$ of incident positrons (so-called $S(E)$-curves) for HT (MaTecK) and PM (Cermet) grown ZnO crystals measured by VEPAS. All $S$-parameters were divided by $S$-parameter of bulk value $S_{0}$ for hydrothermally grown $\mathrm{ZnO}$. At very low energies, virtually all positrons annihilate at surface. With the increasing energy, positrons penetrate deeper and deeper into the sample and the fraction of positrons diffusing back to the surface decreases. This is reflected by a decrease of the $S$-parameter from the surface value to the bulk value corresponding to the situation when all positrons are annihilated in the bulk. Obviously, the HT grown crystal exhibits higher bulk $S$-parameter than the PM grown sample. The $S(E)$ curves were fitted by VEPFIT [16] software package using a single layer model and the model curves calculated by VEPFIT are plotted in Fig. 2 by solid lines. The mean positron diffusion length $L_{+}=47 \pm 1 \mathrm{~nm}$ and $60 \pm 4 \mathrm{~nm}$ was obtained from fitting for HT and PM grown crystal, respectively. These values should be compared with the positron diffusion length $L_{+, \mathrm{B}}$ in a perfect (defect-free) $\mathrm{ZnO}$ crystal.

Since a defect-free $\mathrm{ZnO}$ sample is not available, $L_{+, \mathrm{B}}$ was calculated using the semiclassical random walk the- ory $[17,18]$. Within this approach, the positron diffusion coefficient $D_{+}$in a perfect (defect-free) $\mathrm{ZnO}$ crystal can be determined using the expression

$$
D_{+}=\frac{k T}{m^{*}} \tau_{\mathrm{ph}},
$$

where $k$ is the Boltzmann constant, $T$ is thermodynamical temperature, $m^{*} \approx 1.5 m_{0}$ is the effective positron mass in the units of the rest electron mass $m_{0}$ and $\tau_{\mathrm{ph}}$ is the relaxation time for the positron scattering process. For thermalized positrons in $\mathrm{ZnO}$, the acoustic longitudinal phonon scattering dominates and the relaxation time for this process $\tau_{\mathrm{ph}}$ can be calculated using the deformation-potential approximation [17, 18]:

$$
\tau_{\mathrm{ph}}=\sqrt{\frac{8 \pi}{9}} \frac{\hbar^{4}\left\langle c_{i i}\right\rangle}{\left(m^{*} k T\right)^{\frac{3}{2}} E_{\mathrm{d}}^{2}},
$$

where $\hbar$ is the reduced Planck constant, $\left\langle c_{i i}\right\rangle$ is the averaged elastic constant associated with longitudinal waves [19], and $E_{\mathrm{d}}=3.9 \mathrm{eV}$ is the deformation potential [20]. The positron diffusion coefficient $D_{+}=$ $5.2 \mathrm{~cm}^{2} \mathrm{~s}^{-1}$ was obtained for a perfect $\mathrm{ZnO}$ crystal using Eqs. (1), (2). The mean positron diffusion length in a perfect $\mathrm{ZnO}$ crystal $L_{+, \mathrm{B}}$ can be then calculated as

$$
L_{+, \mathrm{B}}=\sqrt{D_{+} \tau_{\mathrm{B}}} \text {. }
$$

Using $\tau_{\mathrm{B}}=154 \mathrm{ps}$ as bulk positron lifetime, Eq. (3) yields $L_{+, \mathrm{B}}=280 \mathrm{~nm}$. This value is comparable with the positron diffusion lengths measured on well annealed semiconductors which usually fall into the range of 200$300 \mathrm{~nm}[21]$.

Obviously, the positron diffusion lengths determined from fitting of experimental $S(E)$ curves in Fig. 2 are significantly shorter than $L_{+, \mathrm{B}}$. This testifies that both HT and $\mathrm{PM}$ grown $\mathrm{ZnO}$ crystals contain significant concentration of open-volume defects which trap positrons and cause shortening of positron diffusion length. Moreover, the HT grown crystal exhibits shorter positron diffusion length than PM grown sample which indicates that it contains higher density of defects.

From the LT investigations, we know that the positron traps in $\mathrm{ZnO}$ crystals are $\mathrm{Zn}$ vacancies associated with hydrogen and open-volume defects at SFs, i.e. defects with open volume slightly smaller than the zinc vacancy. The concentration $c_{\mathrm{V}}$ of these defects can be estimated from a comparison of the positron diffusion length measured in the sample and that calculated for a perfect $\mathrm{ZnO}$ crystal [22]:

$$
c_{\mathrm{V}}=\frac{1}{\nu_{\mathrm{V}} \tau_{\mathrm{B}}}\left(\frac{L_{+, \mathrm{B}}^{2}}{L_{+}^{2}}-1\right) .
$$

The symbol $\nu_{\mathrm{V}}$ stands for the specific positron trapping rate for cation vacancies. Using $\nu_{\mathrm{V}}=1.7 \times 10^{15} \mathrm{~s}^{-1}$ [21], which is a typical value for a double charge negative vacancy, Eq. (4) yields $c_{\mathrm{V}}=(1.4 \pm 0.1) \times 10^{-4}$ $\left((1.1 \pm 0.1) \times 10^{19} \mathrm{~cm}^{-3}\right)$ and $c_{\mathrm{V}}=(0.8 \pm 0.1) \times 10^{-4}$ $\left((0.7 \pm 0.1) \times 10^{19} \mathrm{~cm}^{-3}\right)$ for the HT and PM grown $\mathrm{ZnO}$ crystal, respectively.

The concentration of defects is given also by the two-state trapping model (STM) [23]: 


$$
c_{\mathrm{V}}=\frac{1}{\nu_{\mathrm{V}}} \frac{I_{2}}{I_{1}}\left(\frac{1}{\tau_{\mathrm{B}}}-\frac{1}{\tau_{\mathrm{D}}}\right)
$$

where $I_{1}$ and $I_{2}$ is the intensity of free positron component and the component which comes from positrons trapped at defects, respectively. The symbol $\tau_{\mathrm{D}}$ denotes the lifetime of trapped positrons, i.e. $\tau_{\mathrm{D}} \approx 180 \mathrm{ps}$ and 165 ps for HT and PM grown $\mathrm{ZnO}$ crystals, respectively. Using Eq. (5) and the defect concentration determined in $\mathrm{ZnO}$ crystals by Eq. (4), one can easily calculate that STM predicts extremely low intensity of the free positron component $I_{1} \leq 1 \%$. In practice, it is not possible to resolve the free positron component when its intensity becomes as small as $\approx 1 \%$. Hence, the density of cation vacancies in $\mathrm{ZnO}$ crystals is so high that it leads to saturated positron trapping which is in accordance with LT results. Measurement of positron diffusion length by VEPAS is more sensitive than LT spectroscopy to variations of defect density in materials where the concentration of defects becomes very high [24]. Hence, VEPAS enables to obtain reliable estimate of defect concentrations in the materials with very high density of defects where LT spectroscopy cannot be used due to saturated trapping.

\section{Conclusions}

An investigation of positron lifetimes for high quality $\mathrm{ZnO}$ single crystals from various suppliers was performed in this work. All $\mathrm{ZnO}$ crystals studied exhibit a single component LT spectrum with lifetime remarkably higher than the bulk $\mathrm{ZnO}$ lifetime due to saturated positron trapping at defects. Two groups of $\mathrm{ZnO}$ crystals can be distinguished: (i) HT grown samples contain zinc vacancies associated with hydrogen and characterized by lifetimes falling in the range 179-182 ps, (ii) crystals grown by other techniques (PM, BG, and VP) contain vacancy-like open volume defects located at SFs and characterized by shorter lifetimes falling in the interval 160-173 ps. This phenomenon can be explained by different nature of the growing methods. In HT method, $\mathrm{ZnO}$ crystals are grown from concentrated aqueous solutions of hydroxides and presence of relatively high amount of hydrogen leads to formation of $\mathrm{V}_{\mathrm{Zn}}-\mathrm{H}$ complexes. $\mathrm{PM}$ and $\mathrm{BG}$ techniques utilizing melting of $\mathrm{ZnO}$ induce stacking faults due to unavoidable temperature gradients in the melt. Similarly, VP growth technique likely introduces SFs into the crystal due to unequal solidification rate. VEPAS investigations revealed that HT grown crystal exhibits higher defect density than PM grown sample.

\section{Acknowledgments}

This work was supported by the Czech Science Agency (project P108/11/0958) and the Charles University in Prague (project SVV-2013-267303).

\section{References}

[1] A. Tsukazaki, A. Ohtomo, T. Onuma, M. Ohtani, T. Makino, M. Sumiya, K. Ohtani, S.F. Chichibu, S. Fuke, Y. Segawa, H. Ohno, H. Koinuma, M. Kawasaki, Nature Mater. 4, 42 (2005).

[2] S. Pearton, D. Norton, K. Ip, Y. Heo, T. Steiner, Prog. Mater. Sci. 50, 293 (2005).
[3] M. McCluskey, S. Jokela, J. Appl. Phys. 106, 071101 (2009).

[4] P. Hautojärvi, Positrons in Solids, Springer-Verlag, Berlin 1979.

[5] F. Tuomisto, D.C. Look, Proc. SPIE 6474, 647413 (2007).

[6] Z.Q. Chen, A. Kawasuso, Y. Xu, H. Naramoto, X.L. Yuan, T. Sekiguchi, R. Suzuki, T. Ohdaira, Phys. Rev. B 71, 115213 (2005).

[7] A. Sarkar, M. Chakrabarti, S.K. Ray, D. Bhowmick, D. Sanyal, J. Phys., Condens. Matter 23, 155801 (2011).

[8] G. Brauer, W. Anwand, D. Grambole, J. Grenzer, W. Skorupa, J. Cížek, J. Kuriplach, I. Procházka, C.C. Ling, C.K. So, D. Schulz, D. Klimm, Phys. Rev. B 79, 115212 (2009).

[9] S. Brunner, W. Puff, A. Balogh, P. Mascher, Mater Sci. Forum 363, 141 (2001).

[10] F. Bečvář, J. Čížek, I. Procházka, J. Janotová, Nucl. Instrum. Methods., Phys. Res. A 539, 372 (2005).

[11] I. Procházka, I. Novotný, F. Bečvář, Mater. Sci. Forum 255-257, 772 (1997).

[12] W. Anwand, H.R. Kissener, G. Brauer, Acta Phys. Pol. A 88, 7 (1995).

[13] H. Takenaka, D.J. Singh, Phys. Rev. B 75, 241102 (2007).

[14] G. Brauer, W. Anwand, W. Skorupa, J. Kuriplach, O. Melikhova, C. Moisson, H. von Wenckstern, H. Schmidt, M. Lorenz, M. Grundmann, Phys. Rev. B 74, 045208 (2006).

[15] W. Anwand, G. Brauer, R.I. Grynszpan, T.E. Cowan, D. Schulz, D. Klimm, J. Ćížek, J. Kuriplach, I. Procházka, C.C. Ling, A.B. Djurisǐć, V. Klemm, G. Schreiber, D. Rafaja, J. Appl. Phys. 109, 063516 (2011).

[16] A. van Veen, H. Schut, M. Clement, J.M.M. de Nijs, A. Kruseman, M.R. Ijpma, Appl. Surf. Sci. 85, 216 (1995).

[17] J. Bardeen, W. Shockley, Phys. Rev. B 80, 72 (1950).

[18] M.J. Puska, R.M. Nieminen, Rev. Mod. Phys. 66, 841 (1994).

[19] K. Sarasamak, S. Limpijumnong, W.R.L. Lambrecht, Phys. Rev. B 82, 035201 (2010).

[20] J. Wrzesinski, D. Fröhlich, Phys. Rev. B 56, 13087 (1997).

[21] R. Krause-Rehberg, H. Leipner, Positron Annihilation in Semiconductors - Defect Studies, Springer, Berlin 1999.

[22] A. van Veen, H. Schut, J. de Vries, R.A. Hakvoort, M.R. Ijpma, AIP Conf. Proc. 218, 83 (1990).

[23] R. West, in: Positrons in Solids, Ed. P. Hautojärvi, Springer-Verlag, Berlin 1979, p. 89.

[24] J. Čížek, F. Lukáč, I. Procházka, R. Kužel, Y. Jirásková, D. Janičkovič, W. Anwand, G. Brauer, Physica B 407, 2659 (2012). 